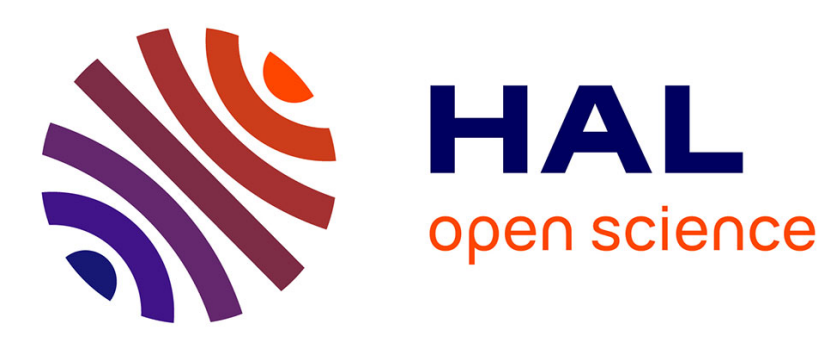

\title{
Measuring Disagreement in Argumentation Graphs
}

\author{
Leila Amgoud, Jonathan Ben-Naim
}

\section{To cite this version:}

Leila Amgoud, Jonathan Ben-Naim. Measuring Disagreement in Argumentation Graphs. 11th International Conference on Scalable Uncertainty Management (SUM 2017), Oct 2017, Grenade, Spain. 10.1007/978-3-319-67582-4_15. hal-02326011

\section{HAL Id: hal-02326011 \\ https://hal.science/hal-02326011}

Submitted on 22 Oct 2019

HAL is a multi-disciplinary open access archive for the deposit and dissemination of scientific research documents, whether they are published or not. The documents may come from teaching and research institutions in France or abroad, or from public or private research centers.
L'archive ouverte pluridisciplinaire HAL, est destinée au dépôt et à la diffusion de documents scientifiques de niveau recherche, publiés ou non, émanant des établissements d'enseignement et de recherche français ou étrangers, des laboratoires publics ou privés. 


\title{
Measuring Disagreement in Argumentation Graphs
}

\author{
Leila Amgoud Jonathan Ben-Naim \\ IRIT - CNRS, France
}

\begin{abstract}
The aim of this paper is to evaluate to what extent an argumentation graph (a set of arguments and attacks between them) is conflicting. For that purpose, we introduce the novel notion of disagreement measure as well as a set of principles that such a measure should satisfy. We propose some intuitive measures and show that they fail to satisfy some of the principles. Then, we come up with a more discriminating measure which satisfies them all. Finally, we relate some measures to those quantifying inconsistency in knowledge bases.
\end{abstract}

\section{Introduction}

An argumentation framework is a graph whose nodes are arguments and edges are attacks between pairs of arguments. The graph may be extracted from a knowledge base (e.g., in [1]), or from a dialogue between agents (e.g., [2]), etc. Whatever the source of the graph, the presence of attacks means existence of disagreements and three questions raise quite naturally: 1) how to model disagreements? 2) what is their amount? and how to solve them? Works in computational argumentation focused mainly on questions 1) and 3). They assume that disagreements in an argumentation graph are nothing more than the attacks of the graph, and represent them either as abstract relations between pairs of arguments (e.g., in [3]), or as logical relations between arguments (e.g., undercut [4], rebuttal [5]). An impressive amount of work has also been done on solving disagreements using the so-called acceptability semantics, of which extension semantics [3] are some examples.

The question of measuring the amount of disagreements in an argumentation graph has never been studied. Consider the six argumentation graphs below. There is no method in the literature that evaluates the amount of disagreement in each of them.

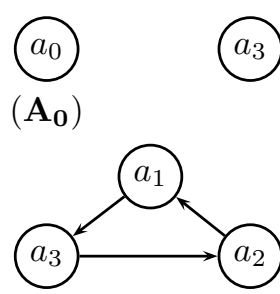

$\left(\mathbf{A}_{3}\right)$

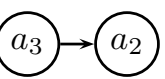

$\left(\mathbf{A}_{1}\right)$

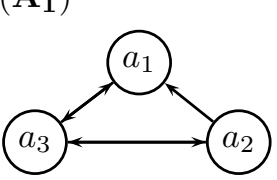

$\left(\mathbf{A}_{4}\right)$

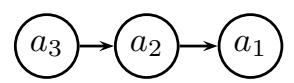

$\left(\mathbf{A}_{2}\right)$

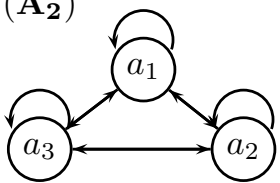

$\left(\mathbf{A}_{5}\right)$

Existing semantics solve disagreements without bothering about their amount. Nevertheless, quantifying disagreements is relevant for various purposes. Namely, in the context of inconsistency handling, an argumentation graph is extracted from a (inconsistent) knowledge base (KB). Quantifying disagreements in the graph allows evaluating 
at what extent the knowledge base is inconsistent. Motivated by important applications like software specifications, quantifying inconsistency in a KB has become a hot topic the last six years (e.g. [6-11]). Since the number of applications of argumentation grows steadily, it is important that the approach has its own tools for answering various needs of the applications including measuring inconsistency. Hence, argumentation not only handles inconsistency in KBs, but it will also be used for measuring inconsistency in those KBs.

The contribution of this paper is fourfold: First, we introduce the novel notion of disagreement measure, that is a real-valued function that assigns to each argumentation framework a value representing its amount of disagreements. Second, we propose principles that a disagreement measure should satisfy. These principles serve as theoretical criteria for judging and comparing disagreement measures. Third, we define five intuitive disagreement measures, one of which satisfies all the principles. Finally, we make a first bridge with works on inconsistency measures by showing that some of our measures return the same result as an existing inconsistency measure.

The paper is structured as follows: Section 2 recalls basic concepts. Section 3 defines disagreement measures and proposes principles they should satisfy. Section 4 introduces six measures and discusses their properties. Section 5 shows how some measures evaluate inconsistency in KBs.

\section{Basic Concepts}

An argumentation framework (or argumentation graph) $\mathbf{A}$ is a graph consisting of a non-empty set $\mathcal{A}$ of nodes representing arguments, and a set $\mathcal{R}$ of links (or edges). A link $r \in \mathcal{R}$ is an ordered pair $\left(a_{1}, a_{2}\right)$ representing a direct attack from argument $a_{1}$ to argument $a_{2}\left(a_{1}, a_{2} \in \mathcal{A}\right)$. Throughout the paper, we write $\mathbf{A}=\langle\mathcal{A}, \mathcal{R}\rangle$. A graph is finite iff its set of arguments is finite.

- A path from argument $a$ to argument $b$ in $\mathbf{A}$ is a sequence $\left\langle a_{0}, \ldots, a_{n}\right\rangle$ of arguments of $\mathcal{A}$ such that $a_{0}=a, a_{n}=b$, for any $0 \leq i<n,\left(a_{i}, a_{i+1}\right) \in \mathcal{R}$, and for all $i \neq j, a_{i} \neq a_{j}$. We say that $b$ is reachable from $a$ when there is a path from $a$ to $b$. If $n=2 m+1$ and $m>0$, then the pair $\left(a_{0}, a_{n}\right)$ is an indirect attack on $a_{n}$.

- A cycle is a path $\left\langle a_{0}, \ldots, a_{n}\right\rangle$ such that $\left(a_{n}, a_{0}\right) \in \mathcal{R}$. It is elementary iff there does not exist a cycle $\left\langle b_{0}, \ldots, b_{m}\right\rangle$ such that $\left\{b_{0}, \ldots, b_{m}\right\} \subset\left\{a_{0}, \ldots, a_{n}\right\}$. A graph is acyclic if it does not contain any cycle.

- An argumentation graph $\mathbf{A}=\left\langle\left\{a_{0}, \ldots, a_{n}\right\}, \mathcal{R}\right\rangle$ is a cycle iff $\mathcal{R}=\left\{\left(a_{i}, a_{i+1}\right) \mid 0 \leq i<n\right\} \cup\left\{\left(a_{n}, a_{0}\right)\right\}$. The graph $\mathbf{A}=\left\langle\left\{a_{0}, \ldots, a_{n}\right\}, \mathcal{R}\right\rangle$ is a simple path iff $\mathcal{R}=\left\{\left(a_{i}, a_{i+1}\right) \mid 0 \leq i<n\right\}$.

- The length of a path (resp. cycle) $\left\langle a_{0}, \ldots, a_{n}\right\rangle$ is $n$ (resp. $n+1$ ).

- An isomorphism from $\mathbf{A}=\langle\mathcal{A}, \mathcal{R}\rangle$ to $\mathbf{A}^{\prime}=\left\langle\mathcal{A}^{\prime}, \mathcal{R}^{\prime}\right\rangle$ is a bijective function $f$ from A to $\mathbf{A}^{\prime}$ such that $\forall a, b \in \mathcal{A},(a, b) \in \mathcal{R}$ iff $(f(a), f(b)) \in \mathcal{R}^{\prime}$.

Notations: We denote by Args an infinite set of all possible arguments, and by $\mathcal{U}$ the universe of finite argumentation graphs built from Args. For any argumentation graph $\mathbf{A}=\langle\mathcal{A}, \mathcal{R}\rangle, \operatorname{Arg}(\mathbf{A})=\mathcal{A}, \operatorname{Att}(\mathbf{A})=\mathcal{R}$, and $\operatorname{SelfAtt}(\mathbf{A})=\{a \in \mathcal{A} \mid(a, a) \in \mathcal{R}\}$. 


\section{Principles for Disagreement Measures}

Our aim is to evaluate the amount of disagreements contained in an argumentation graph. This is done by a disagreement measure, that is a real-valued function that assigns a disagreement value to every argumentation graph.

Definition 1 (Disagreement Measure) $A$ disagreement measure is a function $\mathcal{K}: \mathcal{U} \rightarrow$ $[0,+\infty)$. For an argumentation graph $\mathbf{A}=\langle\mathcal{A}, \mathcal{R}\rangle \in \mathcal{U}, \mathcal{K}(\mathbf{A})$ is called the disagreement value of $\mathbf{A}$.

For two argumentation graphs $\mathbf{A}$ and $\mathbf{A}^{\prime}$, we say that $\mathbf{A}$ is more conflicting than $\mathbf{A}^{\prime}$ if $\mathcal{K}(\mathbf{A})>\mathcal{K}\left(\mathbf{A}^{\prime}\right)$. The value 0 stands for absence of disagreements.

We propose next a set of principles that any disagreement measure should satisfy. The first principle states that the disagreement value of an argumentation graph does not depend on the identity of its arguments. Note that this axiom is used in most axiomatic approaches including game theory (e.g., Shapley value [12]).

Principle 1 (Anonymity) For all argumentation graphs $\mathbf{A}=\langle\mathcal{A}, \mathcal{R}\rangle$ and $\mathbf{A}^{\prime}=\left\langle\mathcal{A}^{\prime}, \mathcal{R}^{\prime}\right\rangle$ in $\mathcal{U}$, if $\mathbf{A}$ and $\mathbf{A}^{\prime}$ are isomorphic, then $\mathcal{K}(\mathbf{A})=\mathcal{K}\left(\mathbf{A}^{\prime}\right)$.

The second principle states that attacks are the only source of disagreements. Thus, any argumentation graph that has an empty attack relation receives the value 0 . This axiom is somehow similar to the consistency axiom proposed in [6] for measuring inconsistency in knowledge bases.

Principle 2 (Agreement) For any argumentation graph $\mathbf{A}=\langle\mathcal{A}, \mathcal{R}\rangle \in \mathcal{U}$, if $\mathcal{R}=\emptyset$, then $\mathcal{K}(\mathbf{A})=0$.

The third principle concerns "harmless" arguments (i.e., arguments which neither attack nor are attacked by other arguments). The principle states that adding such arguments to an argumentation graph will not modify its disagreement value. This axiom is also in the same spirit as the "free formula independence" axiom proposed in [6].

Principle 3 (Dummy) For any argumentation graph $\mathbf{A}=\langle\mathcal{A}, \mathcal{R}\rangle \in \mathcal{U}$, for any a $\in$ Args $\backslash \mathcal{A}, \mathcal{K}(\mathbf{A})=\mathcal{K}\left(\mathbf{A}^{\prime}\right)$, where $\mathbf{A}^{\prime}=\langle\mathcal{A} \cup\{a\}, \mathcal{R}\rangle$.

The next principle states that if new attacks are added to an argumentation graph, its disagreement value increases. This axiom is in the spirit of monotony axiom in [6] which states that if a knowledge base is extended by formulas, its inconsistency degree cannot decrease.

Principle 4 (Monotony) For any argumentation graph $\mathbf{A}=\langle\mathcal{A}, \mathcal{R}\rangle \in \mathcal{U}$, for any $r \in(\mathcal{A} \times \mathcal{A}) \backslash \mathcal{R}, \mathcal{K}(\mathbf{A})<\mathcal{K}\left(\mathbf{A}^{\prime}\right)$, where $\mathbf{A}^{\prime}=\langle\mathcal{A}, \mathcal{R} \cup\{r\}\rangle$.

So far, we have seen that disagreements contained in an argumentation graph are due to direct attacks (i.e., elements of $\mathcal{R}$ ). It is also well-known that the role of such attacks is to weaken their targets (see the weakening property in [13]). Indeed, whatever the semantics that is used for evaluating arguments, it should satisfy the weakening property since it defines the essence of attacks. However, the effect of weakening may propagate in the graph, giving birth to indirect attacks. Consider the following graph. 


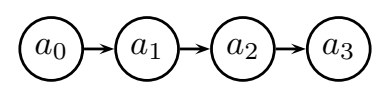

Under stable semantics [3], the graph has one extension $\left\{a_{0}, a_{2}\right\}$, and the argument $a_{3}$ is rejected. If we remove the attack from $a_{0}$ to $a_{1}$, the new graph has $\left\{a_{0}, a_{1}, a_{3}\right\}$ as stable extension, and $a_{3}$ becomes accepted. Thus, the attack $\left(a_{0}, a_{1}\right)$ has a negative effect on $a_{3}$. The same phenomenon occurs under the $h$-categorizer semantics proposed by Besnard and Hunter [1]. The argument $a_{3}$ has an acceptability degree 0.60 in the initial graph and 0.66 in the modified one. Thus, $a_{3}$ looses weight in presence of the attack $\left(a_{0}, a_{1}\right)$. The argument $a_{0}$ is then considered as an indirect attacker of $a_{3}$. This shows that indirect attacks are also source of disagreement in argumentation graphs since they are not only harmful for their direct targets ( $a_{1}$ in the example), but also to the indirect ones $\left(a_{3}\right)$.

The next principle states that an acyclic graph containing indirect attacks is more conflicting than an acyclic graph containing only direct ones. This holds for graphs that have the same number of arguments and the same number of attacks.

Principle 5 (Reinforcement) For argumentation graphs $\mathbf{A}=\langle\mathcal{A}, \mathcal{R}\rangle$ and $\mathbf{A}^{\prime}=\left\langle\mathcal{A}^{\prime}, \mathcal{R}^{\prime}\right\rangle$ in $\mathcal{U}$ such that:

- $\mathcal{A}=\mathcal{A}^{\prime}=\left\{a_{0}, \ldots, a_{n}, b_{0}, \ldots, b_{n}\right\}$ with $n \geq 3$,

- $\mathcal{R}=\left\{\left(a_{i}, b_{i}\right) \mid i \in\{0, \ldots, n-1\}\right\}$,

- $\mathcal{R}^{\prime}=\left\{\left(a_{i}, a_{i+1}\right) \mid i \in\{0, \ldots, n-1\}\right\}$,

it holds that $\mathcal{K}\left(\mathbf{A}^{\prime}\right)>\mathcal{K}(\mathbf{A})$.

The two graphs $\mathbf{A}$ and $\mathbf{A}^{\prime}$ have $n-1$ direct attacks. In addition, $\mathbf{A}^{\prime}$ contains at least one indirect attack (e.g. $\left(a_{0}, a_{n}\right)$ when $\left.n=3\right)$. So, $\mathbf{A}$ is less conflicting than $\mathbf{A}^{\prime}$. Note that due to the Anonymity principle, Reinforcement holds also for argumentation graphs that contain different sets of arguments.

The two argumentation graphs of Reinforcement are acyclic. Assume now an acyclic graph with 100 direct attacks and a 10-length elementary cycle. The latter contains thus 10 attacks and several indirect attacks. Which of the two graphs is more conflicting? There are two possible (but incompatible) answers to this question: i) to give more weight to disagreements generated by direct attacks, ii) to give an overwhelming weight to cycle since it represents a deadlock situation while conflicts are open in an acyclic graph. This second choice is captured by the following optional principle.

Principle 6 (Cycle Precedence) For all graphs $\mathbf{A}=\langle\mathcal{A}, \mathcal{R}\rangle$ and $\mathbf{A}^{\prime}=\left\langle\mathcal{A}^{\prime}, \mathcal{R}^{\prime}\right\rangle$ in $\mathcal{U}$, if $\mathbf{A}$ is acyclic and $\mathbf{A}^{\prime}$ is an elementary cycle, then $\mathcal{K}(\mathbf{A})<\mathcal{K}\left(\mathbf{A}^{\prime}\right)$.

The last and optional principle says that a disagreement measure could take the size of cycles into account. The idea is that the larger the size of a cycle is, the less severe the disagreement; said differently, the less arguments are needed to produce a cycle, the more "obvious" and strong the disagreement. For instance, a cycle of length 2 is more conflicting than a cycle of length 1000 . The latter is less visible than the former. 
Principle 7 (Size Sensitivity) For all elementary cycles $\mathbf{A}=\langle\mathcal{A}, \mathcal{R}\rangle, \mathbf{A}^{\prime}=\left\langle\mathcal{A}^{\prime}, \mathcal{R}^{\prime}\right\rangle$ in $\mathcal{U}$, if $\left|\mathcal{A}^{\prime}\right|<|\mathcal{A}|$, then $\mathcal{K}(\mathbf{A})<\mathcal{K}\left(\mathbf{A}^{\prime}\right)$.

The seven principles are independent (none of them follows from the others). They are also compatible (they can be satisfied all together by a disagreement measure).

Theorem 1 The principles are independent and compatible.

\section{Five Disagreement Measures}

This section introduces disagreement measures and analytically evaluates them against the proposed principles, especially the five mandatory ones. We introduce them from the most naive to the most elaborated one.

\subsection{Connectance Measure}

The first measure that comes in mind for evaluating disagreements in an argumentation graph is the one that counts the number of attacks in a graph. Such a measure is very natural since disagreements come from attacks.

Definition 2 (Connectance measure) Let $\mathbf{A}=\langle\mathcal{A}, \mathcal{R}\rangle$ be an argumentation graph. $\mathcal{K}_{c}(\mathbf{A})=|\mathcal{R}|$.

Let us illustrate the measure with a running example.

Example 1 Consider the six argumentation graphs from the introduction. It can be checked that $\mathcal{K}_{c}\left(\mathbf{A}_{\mathbf{0}}\right)=0, \mathcal{K}_{c}\left(\mathbf{A}_{\mathbf{1}}\right)=1, \mathcal{K}_{c}\left(\mathbf{A}_{\mathbf{2}}\right)=2, \mathcal{K}_{c}\left(\mathbf{A}_{\mathbf{3}}\right)=3, \mathcal{K}_{c}\left(\mathbf{A}_{\mathbf{4}}\right)=5$, and $\mathcal{K}_{c}\left(\mathbf{A}_{\mathbf{5}}\right)=9$.

The measure $\mathcal{K}_{c}$ satisfies four out of seven principles.

Theorem 2 Connectance measure satisfies Anonymity, Agreement, Dummy, and Monotony. It violates Reinforcement, Size sensitivity and Cycle Precedence.

The fact that $\mathcal{K}_{c}$ violates Reinforcement means that it does not take into account indirect attacks, which is a real weakness of a disagreement measure. This shows also that the amount of disagreement is not the simple number of attacks.

\subsection{In-degree Measure}

The second candidate measure counts the number of arguments that are attacked in an argumentation graph.

Definition 3 (In-degree measure) Let $\mathbf{A}=\langle\mathcal{A}, \mathcal{R}\rangle$ be an argumentation graph. $\mathcal{K}_{i}(\mathbf{A})=$ $|\{a \in \mathcal{A} \mid \exists(x, a) \in \mathcal{R}\}|$. 
Let us illustrate the measure with the six graphs given in the introduction.

Example 1 (Cont): According to the In-degree measure, $\mathcal{K}_{i}\left(\mathbf{A}_{\mathbf{0}}\right)=0, \mathcal{K}_{i}\left(\mathbf{A}_{\mathbf{1}}\right)=1$, $\mathcal{K}_{i}\left(\mathbf{A}_{\mathbf{2}}\right)=2$, and $\mathcal{K}_{i}\left(\mathbf{A}_{\mathbf{3}}\right)=\mathcal{K}_{i}\left(\mathbf{A}_{\mathbf{4}}\right)=\mathcal{K}_{i}\left(\mathbf{A}_{\mathbf{5}}\right)=3$. Thus, $\mathbf{A}_{\mathbf{3}}$ is more conflicting than $\mathbf{A}_{\mathbf{2}}$ which is more conflicting than $\mathbf{A}_{\mathbf{1}}$.

This measure satisfies only three out of seven principles.

Theorem 3 In-degree measure satisfies Anonymity, Agreement, and Dummy. It violates Monotony, Reinforcement, Cycle Precedence, and Size Sensitivity.

This measure has two weaknesses: it does not distinguish an elementary cycle from a complete graph (see graphs $\mathbf{A}_{\mathbf{3}}$ and $\mathbf{A}_{\mathbf{5}}$ in Example 1). Moreover, like Connectance measure, it does not take into account indirect attacks.

Remark: In-degree measure focuses on attacked arguments. One may define another measure which rather evaluates the number of "aggressive" arguments, that is, arguments which attack other arguments. Such a measure satisfies (respectively violates) exactly the same principles as In-degree measure. Thus, it is not a good candidate for assessing disagreement in an argumentation graph.

\subsection{Extension-based Measures}

We now define two measures that are based on acceptability semantics, namely on extension-based semantics proposed in [3]. Those semantics were introduced for solving disagreements in an argumentation graph. Before introducing the measures, let us first recall the semantics we will consider. Let $\mathbf{A}=\langle\mathcal{A}, \mathcal{R}\rangle$ be an argumentation graph and $\mathcal{E} \subseteq \mathcal{A}$.

- $\mathcal{E}$ is conflict-free iff $\nexists a, b \in \mathcal{E}$ such that $(a, b) \in \mathcal{R}$.

- $\mathcal{E}$ defends an argument $a \in \mathcal{A}$ iff $\forall b \in \mathcal{A}$, if $(b, a) \in \mathcal{R}$, then $\exists c \in \mathcal{E}$ such that $(c, b) \in \mathcal{R}$.

Definition 4 (Acceptability semantics) Let $\mathbf{A}=\langle\mathcal{A}, \mathcal{R}\rangle$ be an argumentation graph, and $\mathcal{E} \subseteq \mathcal{A}$ be conflict-free.

$-\mathcal{E}$ is a naive extension iff it is a maximal (w.r.t. set $\subseteq$ ) conflict-free set.

$-\mathcal{E}$ is a preferred extension iff it is a maximal (w.r.t. set $\subseteq$ ) set that defends all its elements.

Notations: $\operatorname{Ext}_{x}(\mathbf{A})$ denotes the set of all extensions of $\mathbf{A}$ under semantics $x$ where $x \in\{n, p\}$ and $n$ (respectively $p$ ) stands for naive (respectively preferred).

The basic idea behind extension-based measures is that the existence of multiple extensions means presence of disagreements in the graph. Furthermore, the greater the number of extensions of an argumentation graph, the greater the amount of disagreements in the graph. However, a disagreement measure which counts only the number of extensions (under a given semantics) may miss disagreements. Consider the following argumentation graph: 


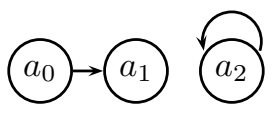

This graph has two naive extensions ( $\left\{a_{0}\right\}$ and $\left\{a_{1}\right\}$ ), which are mainly due to the conflict between $a_{0}$ and $a_{1}$ neglecting thus the self-attack. Similarly, the graph has a single preferred extension $\left\{a_{0}\right\}$ and the self-attack is again neglected. In what follows, we propose two measures (one for each of the two semantics recalled above) which take into account both the number of extensions and the number of self-attacking arguments in an argumentation graph.

Definition 5 (Extension-based measure) Let $\mathbf{A}=\langle\mathcal{A}, \mathcal{R}\rangle$ be an argumentation graph and $x \in\{n, p\}$.

$$
\mathcal{K}_{e}^{x}(\mathbf{A})=\left|\operatorname{Ext}_{x}(\mathbf{A})\right|+|\operatorname{SelfAtt}(\mathbf{A})|-1 .
$$

The subtraction of 1 in the above equation is required in order to ensure agreement in case of empty attack relations.

Example 1 (Cont): Under naive semantics, $\mathcal{K}_{e}^{n}\left(\mathbf{A}_{\mathbf{0}}\right)=0, \mathcal{K}_{e}^{n}\left(\mathbf{A}_{\mathbf{1}}\right)=\mathcal{K}_{e}^{n}\left(\mathbf{A}_{\mathbf{2}}\right)=1$, $\mathcal{K}_{e}^{n}\left(\mathbf{A}_{\mathbf{3}}\right)=\mathcal{K}_{e}^{n}\left(\mathbf{A}_{\mathbf{4}}\right)=2$, and $\mathcal{K}_{e}^{n}\left(\mathbf{A}_{\mathbf{5}}\right)=3$.

Under preferred semantics, $\mathcal{K}_{e}^{p}\left(\mathbf{A}_{\mathbf{0}}\right)=\mathcal{K}_{e}^{p}\left(\mathbf{A}_{\mathbf{1}}\right)=\mathcal{K}_{e}^{p}\left(\mathbf{A}_{\mathbf{2}}\right)=\mathcal{K}_{e}^{p}\left(\mathbf{A}_{\mathbf{3}}\right)=0$, $\mathcal{K}_{e}^{p}\left(\mathbf{A}_{\mathbf{4}}\right)=1, \mathcal{K}_{e}^{p}\left(\mathbf{A}_{\mathbf{5}}\right)=3$.

These two measures are clearly not powerful since they are not discriminating as shown in Example 1. For instance, under preferred semantics, the corresponding measure does not make any difference between graphs with empty attack relations $\left(\mathbf{A}_{\mathbf{0}}\right)$ and those that have one preferred (resp. stable) extension $\left(\mathbf{A}_{\mathbf{1}}\right.$ and $\mathbf{A}_{\mathbf{2}}$ ). The measure is also unable to make a difference between a graph which has one non-empty extension and a graph which has a single empty extension $\left(\mathbf{A}_{\mathbf{2}}\right.$ and $\left.\mathbf{A}_{\mathbf{3}}\right)$. The following result confirms these observations. Indeed, the two measures satisfy only three principles out of seven.

Theorem 4 Extension-based measures satisfy Anonymity, Agreement and Dummy. They violate Monotony, Reinforcement, Cycle Precedence, and Size Sensitivity.

Despite the fact that these measures satisfy (respectively violate) the same principles as $\mathcal{K}_{i}$, they may return different results. Indeed, $\mathcal{K}_{i}$ assigns the same value to $\mathbf{A}_{\mathbf{3}}$ and $\mathbf{A}_{\mathbf{4}}$ while $\mathcal{K}_{e}^{p}$ assigns to them different values. Similarly, $\mathcal{K}_{i}$ assigns different values to $\mathbf{A}_{1}$ and $\mathbf{A}_{2}$ while naive measure assigns to both graphs the same value 1.

Remark: It is worth mentioning that it is possible to define other measures using other extension semantics like complete, stable, semi-stable, etc. However, they will all satisfy the same set of principles as the two discussed above.

\subsection{Distance-based Measure}

The previous disagreement measures are unable to take into account indirect attacks. Our last measure escapes this limitation. It satisfies thus reinforcement as well as all the other principles. The basic idea for capturing indirect attacks (and of course direct 


\begin{tabular}{|l|c|c|c|c|}
\hline & $\mathcal{K}_{e}^{x}$ & $\mathcal{K}_{c}$ & $\mathcal{K}_{i}$ & $\mathcal{K}_{d}$ \\
\hline \hline Anonymity & $\bullet$ & $\bullet$ & $\bullet$ & $\bullet$ \\
\hline Agreement & $\bullet$ & $\bullet$ & $\bullet$ & $\bullet$ \\
\hline Dummy & $\bullet$ & $\bullet$ & $\bullet$ & $\bullet$ \\
\hline Monotony & $\circ$ & $\bullet$ & $\circ$ & $\bullet$ \\
\hline Reinforcement & $\circ$ & $\circ$ & $\circ$ & $\bullet$ \\
\hline Cycle Precedence & $\circ$ & $\circ$ & $\circ$ & $\bullet$ \\
\hline Size Sensitivity & $\circ$ & $\circ$ & $\circ$ & $\bullet$ \\
\hline
\end{tabular}

Table 1. Satisfaction of principles by the measures (the symbol $\bullet$ stands for satisfaction and $\circ$ for violation.)

attacks) is to check the existence of a path between any pair of arguments of an argumentation graph. Since two arguments may be related by several paths, we consider the shortest one. Then, we compute a global distance for the graph which is the sum of the lengths of those paths. Before defining formally the new measure, let us first recall the notion of distance in graphs.

Definition 6 (Distance) Let $\mathbf{A}=\langle\mathcal{A}, \mathcal{R}\rangle$ be an argumentation graph and $a, b \in \mathcal{A}$. If $a \neq b$, then the distance between $a$ and $b$ in $\mathbf{A}, d(a, b)$, is the length of the shortest path from $a$ to $b$ if $b$ is reachable from $a$, and $d(a, b)=k$ if $b$ is not reachable from $a$. If $a=b, d(a, b)$ is the length of the shortest elementary cycle in which a is involved, and $d(a, b)=k$ if $a$ is not involved in cycles. Throughout the paper, we assume that $k=|\mathcal{A}|+1$.

Note that $k$ is set to $|\mathcal{A}|+1$ because the longest path in an argumentation graph is $|\mathcal{A}|-1$ and the length of the longest cycle is $|\mathcal{A}|$.

Example 1 (Cont): In argumentation graph $\mathbf{A}_{\mathbf{3}}, d\left(a_{1}, a_{1}\right)=3, d\left(a_{1}, a_{2}\right)=2$ and $d\left(a_{1}, a_{3}\right)=1$. In graph $\mathbf{A}_{\mathbf{2}}, d\left(a_{1}, a_{1}\right)=4$ and $d\left(a_{1}, a_{3}\right)=4$ (here $\left.k=4\right)$.

The domain of the distance function is delimited as follows.

Proposition 1 Let $\mathbf{A}=\langle\mathcal{A}, \mathcal{R}\rangle$ be an argumentation graph. For all $a, b \in \mathcal{A}, d(a, b) \in$ $[1, k]$.

The global distance of an argumentation graph is the sum of lengths of the shortest paths between any pair of arguments.

Definition 7 (Global distance) For any argumentation graph $\mathbf{A}=\langle\mathcal{A}, \mathcal{R}\rangle$,

$$
\mathcal{D}(\mathbf{A})=\sum_{a_{i} \in \mathcal{A}} \sum_{a_{j} \in \mathcal{A}} d\left(a_{i}, a_{j}\right)
$$

Example 1 (Cont): $\mathcal{D}\left(\mathbf{A}_{\mathbf{0}}\right)=2, \mathcal{D}\left(\mathbf{A}_{\mathbf{1}}\right)=10, \mathcal{D}\left(\mathbf{A}_{\mathbf{2}}\right)=28, \mathcal{D}\left(\mathbf{A}_{\mathbf{3}}\right)=18, \mathcal{D}\left(\mathbf{A}_{\mathbf{4}}\right)=13$ and $\mathcal{D}\left(\mathbf{A}_{\mathbf{5}}\right)=9$.

Let us now delimit the upper and lower bounds of the global distance of an argumentation graph. 
Proposition 2 For any argumentation graph $\mathbf{A}=\langle\mathcal{A}, \mathcal{R}\rangle$,

$$
\min \leq \mathcal{D}(\mathbf{A}) \leq \max
$$

where $\max =n^{2} \times(n+1), \min =n^{2}$ and $n=|\mathcal{A}|$.

We show next that the upper bound is reached by an argumentation graph in case its attack relation is empty, while the lower bound is reached when the graph is complete.

Proposition 3 For any argumentation graph $\mathbf{A}=\langle\mathcal{A}, \mathcal{R}\rangle$,

- $\mathcal{D}(\mathbf{A})=\max$ iff $\mathcal{R}=\emptyset$

- $\mathcal{D}(\mathbf{A})=\min$ iff $\mathcal{R}=\mathcal{A} \times \mathcal{A}$

Distance-based measure evaluates to what extent the global distance of an argumentation graph is close to the upper bound. The more it is close to max, the less disagreements are in the graph. The closer the global distance is to min, the more the graph contains a lot of conflicts.

Definition 8 (Distance-based measure) For any argumentation graph $\mathbf{A}=\langle\mathcal{A}, \mathcal{R}\rangle$,

$$
\mathcal{K}_{d}(\mathbf{A})=\frac{\max -\mathcal{D}(\mathbf{A})}{\max -\min }
$$

where $\max =n^{2} \times(n+1)$, $\min =n^{2}$ and $n=|\mathcal{A}|$.

Let us illustrate this measure with the running example.

Example 1 (Cont): $\mathcal{K}_{d}\left(\mathbf{A}_{\mathbf{0}}\right)=0, \mathcal{K}_{d}\left(\mathbf{A}_{\mathbf{1}}\right)=0.25, \mathcal{K}_{d}\left(\mathbf{A}_{\mathbf{2}}\right)=0.29, \mathcal{K}_{d}\left(\mathbf{A}_{\mathbf{3}}\right)=0.66$, $\mathcal{K}_{d}\left(\mathbf{A}_{\mathbf{4}}\right)=0.88$, and $\mathcal{K}_{d}\left(\mathbf{A}_{\mathbf{5}}\right)=1$.

This measure computes somehow the degree of connectivity of an argumentation graph. Indeed, a high disagreement value means that the graph is highly connected, and small disagreement value means that the graph is not very connected. It makes thus fine grained comparisons of argumentation graphs, namely of various forms of cyclic graphs. In Example 1, $\mathbf{A}_{5}$ is more conflicting than $\mathbf{A}_{\mathbf{4}}$ which is itself more conflicting than $\mathbf{A}_{3}$.

In what follows, we introduce the notion of connectivity degree of an argumentation graph. It is the proportion of pairs of arguments which are related by at least one path.

Definition 9 (Connectivity degree) The connectivity degree of an argumentation graph $\mathbf{A}=\langle\mathcal{A}, \mathcal{R}\rangle$ is

$$
\operatorname{Co}(\mathbf{A})=\frac{\left|\left\{(a, b) \in \mathcal{A}^{2} \mid d(a, b)<k\right\}\right|}{|\mathcal{A}|^{2}} .
$$

The next result shows that the upper bound of the disagreement value of an argumentation graph is exactly the connectivity degree of the graph.

Theorem 5 For any argumentation graph $\mathbf{A}=\langle\mathcal{A}, \mathcal{R}\rangle, \mathcal{K}_{d}(\mathbf{A}) \in[0, \operatorname{Co}(\mathbf{A})]$. 
Proof. Let $\mathbf{A}=\langle\mathcal{A}, \mathcal{R}\rangle$ be an argumentation graph such that $|\mathcal{A}|=n$. Let $\mathcal{B}=$ $\left\{\left(a_{i}, a_{j}\right) \in \mathcal{A} \times \mathcal{A} \mid d\left(a_{i}, a_{j}\right)<k\right\}$. From Proposition ??, $\mathcal{K}_{d}(\mathbf{A})=\operatorname{Co}(\mathbf{A})+\frac{\operatorname{Co}(\mathbf{A})}{n}-$ $\frac{\sum_{\left(a_{i}, a_{j}\right) \in \mathcal{B}} d\left(a_{i}, a_{j}\right)}{n^{3}}=\frac{\operatorname{Co}(\mathbf{A})(n+1)}{n}-\frac{\sum_{\left(a_{i}, a_{j}\right) \in \mathcal{B}} d\left(a_{i}, a_{j}\right)}{n^{3}}$. It holds that $\sum_{\left(a_{i}, a_{j}\right) \in \mathcal{B}} d\left(a_{i}, a_{j}\right) \geq$ $n^{2} \operatorname{Co}(\mathbf{A})$ (since $|\mathcal{B}|=n^{2} \operatorname{Co}(\mathbf{A}$ and $d(a, b) \in[1, k])$. Thus, $\frac{\sum_{\left(a_{i}, a_{j}\right) \in \mathcal{B}} d\left(a_{i}, a_{j}\right)}{n^{3}} \geq$ $\frac{n^{2} \mathrm{Co}(\mathbf{A})}{n^{3}}$. Consequently, $\frac{\operatorname{Co}(\mathbf{A})}{n}-\frac{\sum_{\left(a_{i}, a_{j}\right) \in \mathcal{B}} d\left(a_{i}, a_{j}\right)}{n^{3}} \leq 0$. So, $\mathrm{Co}(\mathbf{A})+\frac{\operatorname{Co}(\mathbf{A})}{n}-\frac{\sum_{\left(a_{i}, a_{j}\right) \in \mathcal{B}} d\left(a_{i}, a_{j}\right)}{n^{3}} \leq$ $\operatorname{Co}(\mathbf{A})$. Thus, $\mathcal{K}_{d}(\mathbf{A}) \leq \mathrm{Co}(\mathbf{A})$.

Let us now characterize the disagreement values of elementary cycles. The shorter an elementary cycle, the more conflicting it is. The maximal value (1) is given to cycles of length 1 , that is graphs that contain only one argument, moreover it is self-attacking. This value decreases when the length of cycles increases. However, we show that it cannot be less than 0.5 . This means that the distance-based measure considers cycles as very conflicting even when they are very long, which is very natural.

Proposition 4 For any elementary cycle $\mathbf{A}=\langle\mathcal{A}, \mathcal{R}\rangle, \mathcal{K}_{d}(\mathbf{A}) \in\left(\frac{1}{2}, 1\right]$.

Proof. Let $\mathbf{A}=\langle\mathcal{A}, \mathcal{R}\rangle$ be an elementary cycle, and let $n=|\mathcal{A}|$. For any $a \in \mathcal{A}$, $\sum_{b_{i} \in \mathcal{A}} d\left(a, b_{i}\right)=1+2+3+\ldots+n=\frac{n \times(n+1)}{2}$. Thus, $\mathcal{D}(\mathbf{A})=\frac{n^{2} \times(n+1)}{2} \cdot \mathcal{K}_{d}(\mathbf{A})=$ $\frac{n^{2} \times(n+1)-\mathcal{D}(\mathbf{A})}{n^{2} \times(n+1)-n^{2}}$, thus $\mathcal{K}_{d}(\mathbf{A})=\frac{n^{2} \times(n+1)}{2 n^{3}}=\frac{n+1}{2 n}=\frac{1}{2}+\frac{1}{2 n} \cdot \mathcal{K}_{d}(\mathbf{A})=1$ in case $n=1$, i.e., $\mathbf{A}$ is made of a self attacking argument. Since $\mathbf{A}$ is finite, then $\mathcal{A}$ is finite. Consequently, $\mathcal{K}_{d}(\mathbf{A})>\frac{1}{2}$.

The next result delimits the disagreement values of acyclic argumentation graphs.

Proposition 5 For any acyclic argumentation graph $\mathbf{A}=\langle\mathcal{A}, \mathcal{R}\rangle, \mathcal{K}_{d}(\mathbf{A}) \in\left[0, \frac{1}{2}\right)$

Proof. Let $\mathbf{A}=\langle\mathcal{A}, \mathcal{R}\rangle$ be an acyclic argumentation graph such that $|\mathcal{A}|=n$. Since A is acyclic, then $\forall a \in \mathcal{A}, d(a, a)=k$. Moreover, $\forall a, b \in \mathcal{A}$, if $d(a, b)<k$ then $d(b, a)=k$ (since there is no cycle in the graph). Thus, $\left|\left\{(a, b) \in \mathcal{A}^{2} \mid d(a, b)<k\right\}\right| \leq$ $\frac{n^{2}-n}{2}$. Consequently, $\frac{\left|\left\{(a, b) \in \mathcal{A}^{2} \mid d(a, b)<k\right\}\right|}{n^{2}} \leq \frac{n^{2}-n}{2 n^{2}}$. We get $\operatorname{Co}(\mathbf{A}) \leq \frac{1}{2}-\frac{1}{2 n}$. Since $\mathcal{A}$ is finite, then $\mathrm{Co}(\mathbf{A})<\frac{1}{2}$. From Theorem $5, \mathcal{K}_{d}(\mathbf{A}) \leq \mathrm{Co}(\mathbf{A})$. So, $\mathcal{K}_{d}(\mathbf{A})<\frac{1}{2}$.

The two previous results show that the measure $\mathcal{K}_{d}$ considers any acyclic graph as strictly less conflicting than any elementary cycle. Moreover, the ratio of disagreement in an acyclic graph is always not very high and can never reach the maximal value 1 . On the contrary, the ratio of disagreement in an elementary cycle is always high.

Proposition 6 Let $\mathbf{A}=\langle\mathcal{A}, \mathcal{R}\rangle$ and $\mathbf{A}^{\prime}=\left\langle\mathcal{A}^{\prime}, \mathcal{R}^{\prime}\right\rangle$ be simple paths. If $|\mathcal{A}|<\left|\mathcal{A}^{\prime}\right|$, then $\mathcal{K}_{d}(\mathbf{A})<\mathcal{K}_{d}\left(\mathbf{A}^{\prime}\right)$.

Proof. Let $\mathbf{A}=\langle\mathcal{A}, \mathcal{R}\rangle$ and $\mathbf{A}^{\prime}=\left\langle\mathcal{A}^{\prime}, \mathcal{R}^{\prime}\right\rangle$ be two simple paths. Let $n=|\mathcal{A}|$ and $n^{\prime}=\left|\mathcal{A}^{\prime}\right|$. Assume that $n<n^{\prime}$. Thus, $n^{2}<n^{\prime 2}$ and $\frac{1}{n^{2}}>\frac{1}{n^{\prime 2}}$. Consequently, $1-\frac{1}{n^{2}}<$ $1-\frac{1}{n^{\prime 2}}$ and then $\mathcal{K}_{d}(\mathbf{A})<\mathcal{K}_{d}\left(\mathbf{A}^{\prime}\right)$.

The distance-based measure satisfies all our principles. 
Theorem $6 \mathcal{K}_{d}$ satisfies all the seven principles.

Proof. Let $\mathbf{A}=\langle\mathcal{A}, \mathcal{R}\rangle$ be an argumentation graph. Anonymity is obviously satisfied. From Prop. 3, if $\mathcal{R}=\emptyset, \mathcal{D}(\mathbf{A})=\max$, thus $\mathcal{K}_{d}(\mathbf{A})=0$ which ensures Agreement.

Let $\mathbf{A}=\langle\mathcal{A}, \mathcal{R}\rangle$ and $\mathbf{A}^{\prime}=\left\langle\mathcal{A}^{\prime}, \mathcal{R}^{\prime}\right\rangle$ be two elementary cycles such that $|\mathcal{A}|=$ $n,\left|\mathcal{A}^{\prime}\right|=m$ and $m>n . \mathcal{D}(\mathbf{A})=\frac{n^{2}(n+1)}{2}$, thus $\mathcal{K}_{d}(\mathbf{A})=\frac{n+1}{2 n}$, and $\mathcal{D}\left(\mathbf{A}^{\prime}\right)=$ $\frac{m^{2}(m+1)}{2}$ and $\mathcal{K}_{d}\left(\mathbf{A}^{\prime}\right)=\frac{m+1}{2 m}=\frac{1}{2}+\frac{1}{2 m}$. Since $m>n$ then $2 m>2 n$ and $\frac{1}{2 m}<\frac{1}{2 n}$. Consequently, $\mathcal{K}_{d}\left(\mathbf{A}^{\prime}\right)<\mathcal{K}_{d}(\mathbf{A})$. This shows that Size Sensitivity is satisfied.

Let us now show that $\mathcal{K}_{d}$ satisfies Dummy principle. Assume that $|\mathcal{A}|=n$ Let $a \in$ $\operatorname{Args} \backslash \mathcal{A}$ and $\mathbf{A}^{\prime}=\langle\mathcal{A} \cup\{a\}, \mathcal{R}\rangle$. We denote by $k$ the maximal distance in graph $\mathbf{A}$ and by $k^{\prime}$ the maximal distance in graph $\mathbf{A}^{\prime}$. From definition, $k^{\prime}=n+2$ since $\left|\operatorname{Arg}\left(\mathbf{A}^{\prime}\right)\right|=$ $n+1$. Since the new arguments does not attack and is not attacked by other arguments, then the original distances in graph $\mathbf{A}$ will not change except those that got value $k$ which will be incremented by 1 each. Thus, $\mathcal{D}\left(\mathbf{A}^{\prime}=\mathcal{D}(\mathbf{A})+(2 n+1) k^{\prime}+x\right.$ where $x \geq 0$ is the number of pairs $\left(a_{i}, a_{j}\right)$ of arguments for which the length of the shortest path from $a_{i}$ to $a_{j}$ is equal to $k$ in graph $\mathbf{A}$. We get $\mathcal{D}\left(\mathbf{A}^{\prime}=\mathcal{D}(\mathbf{A})+2 n^{2}+5 n+2+x\right.$. Moreover, $\mathcal{K}_{d}(\mathbf{A})=1+\frac{1}{n}-\frac{\mathcal{D}(\mathbf{A})}{n^{3}}$ and $\mathcal{K}_{d}\left(\mathbf{A}^{\prime}\right)=1+\frac{1}{n+1}-\frac{\mathcal{D}(\mathbf{A})}{(n+1)^{3}}-\frac{x+2 n^{2}+5 n+2}{(n+1)^{3}}$. Thus, $\mathcal{K}_{d}\left(\mathbf{A}^{\prime}\right)<\mathcal{K}_{d}(\mathbf{A})$.

Let us now show that monotony is also satisfied. Let $\mathcal{R}^{\prime} \subseteq(\mathcal{A} \times \mathcal{A}) \backslash \mathcal{R}$ and $\mathbf{A}^{\prime}=\left\langle\mathcal{A}, \mathcal{R} \cup \mathcal{R}^{\prime}\right\rangle$. Both $\mathbf{A}$ and $\mathbf{A}^{\prime}$ have the same min and max distances since they have the same number of arguments. Consequently, $\mathcal{K}_{d}(\mathbf{A})=1+\frac{1}{n}-\frac{D(\mathbf{A})}{n^{3}}$ and $\mathcal{K}_{d}\left(\mathbf{A}^{\prime}\right)=1+\frac{1}{n}-\frac{D\left(\mathbf{A}^{\prime}\right)}{n^{3}}$, with $n=|\mathcal{A}|$. Assume that $D\left(\mathbf{A}^{\prime}\right)>D(\mathbf{A})$. This means that there exists $a, b \in \mathcal{A}$ such $d(a, b)=x$ in graph $\mathbf{A}, d(a, b)=y$ in graph $\mathbf{A}^{\prime}$ and $y>x$. This is impossible since the shortest path in $\mathbf{A}$ between $a$ and $b$ still exists in $\mathbf{A}^{\prime}$. Thus, in $\mathbf{A}^{\prime}$, the shortest path between $a$ and $b$ is either the same as in $\mathbf{A}$ or a path with $y<x$ because of the additional attacks of $\mathcal{R}^{\prime}$.

Cycle Precedence follows from Propositions 4 and 5.

Reinforcement is also satisfied. Since the two graphs in the principle are assumed to have the same number of arguments, then both graphs have the same max and min values. It is thus sufficient to compare the global distances of the graphs. We can easily compute the following values: $\mathcal{D}(\mathbf{A})=8 n^{3}+26 n^{2}+32 n+14$, and $\mathcal{D}\left(\mathbf{A}^{\prime}\right) \frac{20}{3} n^{3}+$ $25 n^{2}+\frac{91}{3} n+12 . \mathcal{D}(\mathbf{A})>\mathcal{D}\left(\mathbf{A}^{\prime}\right)$, thus $\mathcal{K}(\mathbf{A})<\mathcal{K}\left(\mathbf{A}^{\prime}\right)$.

Distance-based measure satisfies all the principles. Thus, it takes into account both the direct attacks in an argumentation graph as well as the indirect ones. All these features make it the best candidate for measuring disagreements in argumentation graphs. Table 1 recalls for each measure, the principles it satisfies and those it violates.

\section{Links between Disagreement Measures and Inconsistency Measures}

In this section, we consider argumentation graphs $\langle\mathcal{A}, \mathcal{R}\rangle$ that are generated from a propositional knowledge base $\Sigma$. The arguments of $\mathcal{A}$ are defined as follows: 
Definition 10 (Argument) Let $\Sigma$ be a propositional knowledge base. An argument is a pair $(X, x)$ s.t. $X \subseteq \Sigma, X$ is consistent, $X \vdash x^{1}$, and $\nexists X^{\prime} \subset X$ such that $X^{\prime} \vdash x$.

Regarding the attack relation $\mathcal{R}$, we consider assumption-attack defined in [5].

Definition 11 (Assumption-Attack) An argument $(X, x) \operatorname{attacks}(Y, y)(((X, x),(Y, y)) \in$ R) iff $\exists y^{\prime} \in Y$ such that $x \equiv \neg y^{\prime 2}$.

In [14], the authors proposed a measure $(\mathcal{I})$ that quantifies the amount of inconsistency in a propositional knowledge base $\Sigma$. That amount is equal to the number of maximal (for set inclusion) consistent subsets of $\Sigma$ and the number of inconsistent formulas in $\Sigma$ minus 1.

Definition 12 (Inconsistency Measure) For any propositional knowledge base $\Sigma$,

$$
\mathcal{I}(\Sigma)=|\operatorname{Max}(\Sigma)|+|\operatorname{Inc}(\Sigma)|-1
$$

$\operatorname{Max}(\Sigma)$ is the set of maximal (for set $\subseteq$ ) consistent subsets of $\Sigma$, and $\operatorname{Inc}(\Sigma)$ is the set of inconsistent formulae in $\Sigma . \mathcal{I}(\Sigma)$ is called the inconsistency value of $\Sigma$.

Given a knowledge base $\Sigma$, we show that its inconsistency value (as computed by measure $\mathcal{I}$ ) is equal to the disagreement values of the corresponding argumentation graph using the three extension-based measures $\mathcal{K}_{e}^{x}$ with $x \in\{n, p\}$.

Theorem 7 Let $\Sigma$ be a propositional knowledge base such that $\operatorname{Inc}(\Sigma)=\emptyset$. Let $\mathbf{A}=$ $\langle\mathcal{A}, \mathcal{R}\rangle$ be the argumentation graph built over $\Sigma$. The following holds:

$$
\mathcal{K}_{e}^{n}(\mathbf{A})=\mathcal{K}_{e}^{p}(\mathbf{A})=\mathcal{I}(\Sigma)
$$

Proof. Let $\Sigma$ be a propositional knowledge base such that $\operatorname{Inc}(\Sigma)=\emptyset$. Let $\mathbf{A}=$ $\langle\mathcal{A}, \mathcal{R}\rangle$ be the argumentation graph built over $\Sigma$. From Theorem 8 in [15], $\operatorname{Ext}_{\mathrm{n}}(\mathbf{A})=$ $\operatorname{Ext}_{\mathrm{p}}(\mathbf{A})$. Furthermore, there is a full correspondence between the naive extensions of A and the maximal (for set inclusion) consistent subsets of $\Sigma$. Hence, $\left|\operatorname{Ext}_{\mathrm{n}}(\mathbf{A})\right|=$ $|\max (\Sigma)|$. Since $\operatorname{Inc}(\Sigma)=\emptyset$, then $\mathcal{I}(\Sigma)=|\max (\Sigma)|-1$. Since by definition of arguments, $\operatorname{SelfAtt}(\mathbf{A})=\emptyset$, then $\mathcal{K}_{e}^{x}(\mathbf{A})=\left|\operatorname{Ext}_{\mathbf{x}}(\mathbf{A})\right|-1$. Thus, $\mathcal{K}_{e}^{x}(\mathbf{A})=\mathcal{I}(\Sigma)$.

This result shows that not only the two extension-based measures return the same result in case of propositional knowledge bases, but also they are equivalent to the inconsistency measure proposed in [14].

\section{Related work}

Despite the great amount of work on argumentation, there is no work on computing the amount of disagreements in argumentation graphs. Our paper presented the first attempt in this direction. In [16], the authors studied to what extent the extensions (under a given

\footnotetext{
${ }^{1}$ the symbol $\vdash$ stands for propositional inference relation.

${ }^{2}$ The symbol $\equiv$ stands for logical equivalence.
} 
semantics) of an argumentation graph are different. The problem they addressed is thus completely different from the purpose of our paper.

Several measures were proposed in the literature for quantifying inconsistency in propositional knowledge bases (e.g., [7, 6, 8]). Our extension-based measures are equivalent to one of them, namely the one proposed in [14].

\section{Conclusion}

This paper studied for the first time how to quantify disagreements in an argumentation graph. It showed that disagreements is more than direct attacks. It proposed principles which serve as theoretical criteria for validating and comparing disagreement measures. It defined six intuitive measures and investigated their properties. The distance-based measure is the most powerful one. It not only satisfies all the proposed principles, but it is also very discriminating, that is, it provides a fine grained evaluation of argumentation graphs. Moreover, it captures very well the two sources of disagreement: direct and indirect attacks. Furthermore, the paper made a first bridge with works on inconsistency measures. It showed that extension-based measures return the same amount of conflict as one proposed in [14].

This work can be extended in several ways. First, we plan to investigate more deeply the relationship between the disagreement value of an argumentation graph and existing inconsistency degree of the knowledge base over which the graph is built. A particular focus will be put on distance-based measure since it captures well indirect attacks in an argumentation graph. Another line of research consists of evaluating the contribution of each argument to the disagreement value of a graph. Such information may be useful in a dialogues for identifying the culprit that should be attacked.

\section{Acknowledgments}

This work was supported by ANR-13-BS02-0004 and ANR-11-LABX-0040-CIMI.

\section{References}

1. Besnard, P., Hunter, A.: A logic-based theory of deductive arguments. Artificial Intelligence Journal 128 (1-2) (2001) 203-235

2. Bonzon, E., Maudet, N.: On the outcomes of multiparty persuasion. In: 10th International Conference on Autonomous Agents and Multiagent Systems (AAMAS 2011). (2011) 47-54

3. Dung, P.M.: On the acceptability of arguments and its fundamental role in nonmonotonic reasoning, logic programming and n-person games. Artif. Intell. 77(2) (1995) 321-358

4. Pollock, J.: How to reason defeasibly. Artificial Intelligence 57(1) (1992) 1-42

5. Elvang-Gøransson, M., Fox, J., Krause, P.: Acceptability of arguments as 'logical uncertainty'. In: 2nd European Conference on Symbolic and Quantitative Approaches to Reasoning and Uncertainty (ECSQARU'93). Volume 747 of Lecture Notes in Computer Science., Granada, Spain, Springer (November 1993) 85-90

6. Hunter, A., Konieczny, S.: On the measure of conflicts: Shapley inconsistency values. Artificial Intelligence 174(14) (2010) 1007-1026 
7. Grant, J., Hunter, A.: Distance-based measures of inconsistency. In: Proceedings of the European Conference on Symbolic and Quantitative Approaches to Reasoning with Uncertainty (ECSQARU'13). (2013) 230-241

8. Jabbour, S., Ma, Y., Raddaoui, B.: Inconsistency measurement thanks to mus decomposition. In: International conference on Autonomous Agents and Multi-Agent Systems,AAMAS '14. (2014) 877-884

9. Thimm, M.: Towards large-scale inconsistency measurement. In: KI 2014: Advances in Artificial Intelligence - Proceedings of the 37th Annual German Conference on AI. (2014) 195-206

10. Jabbour, S., Ma, Y., Raddaoui, B., Sais, L., Salhi, Y.: On structure-based inconsistency measures and their computations via closed set packing. In: Proceedings of the 2015 International Conference on Autonomous Agents and Multiagent Systems, AAMAS 2015. (2015) $1749-1750$

11. Jabbour, S., Sais, L.: Exploiting MUS structure to measure inconsistency of knowledge bases. In: 22nd European Conference on Artificial Intelligence, ECAI 2016. (2016) 991998

12. Shapley, L.: A values for n-person games. H.W. Kuhn and A.W. Tucker, eds., Contributions to the theory of games, vol. II. Annal of Mathematics Studies (28) (1953)

13. Amgoud, L., Ben-Naim, J.: Axiomatic foundations of acceptability semantics. In: Proceedings of the Fifteenth International Conference Principles of Knowledge Representation and Reasoning, KR 2016. (2016) 2-11

14. Grant, J., Hunter, A.: Measuring consistency gain and information loss in stepwise inconsistency resolution. In: Symbolic and Quantitative Approaches to Reasoning with Uncertainty - 11th European Conference. (2011) 362-373

15. Amgoud, L., Besnard, P.: Logical limits of abstract argumentation frameworks. Journal of Applied Non-Classical Logics 23(3) (2013) 229-267

16. Booth, R., Caminada, M., Podlaszewski, M., Rahwan, I.: Quantifying disagreement in argument-based reasoning. In: International Conference on Autonomous Agents and Multiagent Systems, AAMAS 2012. (2012) 493-500 